\title{
Pharmaco-economic and clinical impact of pre-analytical non conformities in cyclosporin therapeutic drug monitoring
}

\author{
Mehdi Bouhlel, Emna Gaies, Rim Charfi, Issam Salouage, Nadia Jebabli, Mouna Ben Sassi, \\ Hanene El Jebari, Anis Klouz, Raidh Daghfous, Sameh Trabelsi
}

Clinical Pharmacology Department, National Centre of Pharmacovigilance, Tunisia

Background:

Pre-analytical phase is an important step that conditions the quality of laboratory tests. Errors may concern requested test, sampling, identification of patient and sample conservation.

In laboratories of therapeutic drug monitoring (TDM), errors in pre-analytical phase may concern the modalities of sample withdrawal including the appropriate time (trough concentration or pic concentration) and / or the time to reach the steady state. A failure in respecting this timing will lead to incorrect results.

The aim of our work is to assess the pharmaco-economic and pharmaco-clinical impact of non-respecting the time to reach the steady state of cyclosporin (CsA) for TDM of this drug in adult bone marrow transplant patients.

Methods:

It is a retrospective study including adult bone marrow transplant patients during the first month of using CsA.

Trough blood concentration of $\mathrm{CsA}(\mathrm{CsA} \mathrm{C} 0)$ was measured using an immuno-enzymatic technique. The therapeutic interval (TI) was [150-300 ng.mL-1]. Our patients were divided into three groups: group within the TI, group under the $\mathrm{TI}$ and group above the TI.

From all patients, we have calculated the rate of samples not respecting the time to reach the steady state and then we have deduced the cost of the determination of blood concentration of the none conform samples based on the cost of one sample treatment in our laboratory.

Results:

We have collected 1387 samples from 77 patients. We have found 255 (18.38\%) non conformities concerning time to reach the steady state. The global cost was 13000 Tunisian Dinars, equivalent to 270 sample tests.

Results of non-valid CsA C0 were within therapeutic range (TR) in 14 patients (18.2\%), above TR in 12 patients (15.6\%) and under TR in 51 patients (66.2\%).

Conclusion:

Pre-analytical non-conformities can lead to a wrong dose adjustment with a risk of toxicity or inefficiency. In our study the dose adjustment may be made in $81.8 \%$ of patients having non valid CsA concentration. So, establishment of recommendations would be useful in order to harmonize the practices of medical and paramedical staff. Also, this affects the public health economics through highly increasing the cost of the treatment of patients. 